\title{
Philosophiques
}

\section{Modernité des Grecs}

L'importance de la pensée grecque et les raisons de l'enseigner dans le programme collégial de philosophie

\section{Georges Leroux}

Volume 24, numéro 1, printemps 1997

Avez-vous lu Rawls?

URI : https://id.erudit.org/iderudit/027428ar

DOI : https://doi.org/10.7202/027428ar

Aller au sommaire du numéro

Éditeur(s)

Société de philosophie du Québec

ISSN

0316-2923 (imprimé)

1492-1391 (numérique)

Découvrir la revue

Citer cet article

Leroux, G. (1997). Modernité des Grecs : l'importance de la pensée grecque et les raisons de l'enseigner dans le programme collégial de philosophie.

Philosophiques, 24(1), 141-160. https://doi.org/10.7202/027428ar d'utilisation que vous pouvez consulter en ligne.

https://apropos.erudit.org/fr/usagers/politique-dutilisation/ 


\title{
MODERNITÉ DES GRECS \\ I'MPORTANCE DE LA PENSEE GRECQUEF ET IFS RAISONS DE I'ENSELGNER DANS LE PROGRAMME COLIEGGHL DE PHILOSOPHIE*
}

\author{
PAR \\ George \& LerouX
}

Parler de l'actualité d'une pensée, c'est accepter de considérer qu'une pensée pourrait cesser d'être actuelle, qu'elle pourrait s'effacer dans une indifférence à laquelle d'autres urgences la condamneraient. Que cette question puisse se poser concernant la pensée grecque n'a rien d'improbable, tant les raisons qui ont maintenue cette pensée vivante durant des siècles sont devenues avec le temps des habitudes. Combien de générations passées ont été formées dans l'amour et la vénération de la pensée grecque, sans que jamais il ait été besoin de produire une justification des enseignements qui leur ètaient prodigués? Dans la tourmente actuelle, alors que rien ne parait stable dans le cour des curriculums d'étude, ces habitudes sont mises en question. Pourquoi donc l'étude de la philosophie s'amorcerait-elle nécessairement à compter d'un retour aux Grecs? En fait, les arguments soutenant la légitimité et la fécondité de l'étude de la source grecque ont longtemps été considérés comme universels et définitifs, ils avaient êté ènoncés à la Renaissance et l'humanisme classique, qui s'est trouvé à la base des curriculums des collèges européens et américains depuis le début du siècle, n'a jamais remis en question l'héritage de la tradition des humanités. Les choses ont changé et cette position mérite qu'on l'examine de nouveau en fonction des exigences de notre temps. Actualiser la pensée grecque ne peut plus seulement signifier le devoir de recueillir l'héritage grec, ou encore de retourner à la source traditionnelle d'une culture ; actualiser signifie trouver des raisons de garder vivante cette pensée, et chercher à illustrer la forme présente de son actualité pour en justifier l'étude.

Sur le fond de cette longue tradition de l'humanisme, il pourrait sembler entièrement naturel, au moment de consolider la réforme de l'enseignement collégial de la philosophie, de donner une place centrale à la pensée grecque dans le premier ensemble, consacré à l'apprentissage de l'argumentation et à l'étude de l'avènement de la rationalité. Ce n'est pourtant pas le cas. Je voudrais évoquer à cet égard une rencontre avec un collègue du cégep Ahuntsic, LaurentMichel Vacher, qui malgré sa brièveté a laissé en moi beaucoup de questions. Alors que je louais cette orientation vers la pensée grecque, il me surprit entièrement en me disant que cette position était

* Conférence prononcée au Comité des enseignants de philosophie le 26 avril 1996, à Guêbec. 
complètement rétrograde, pour ne pas dire réactionnaire : le retour à la pensée grecque n'était selon lui que l'expression d'un réflexe de sécurité dans un monde en pleine explosion épistémologique et scientifique, une sorte de repli sur des bases en apparence solides, mais qui ne représentaient en réalité que la sécurité d'une tradition. Ce repli manifesterait selon lui deux carences fondamentales : premièrement, une réaction de fuite devant les exigences logicomathématiques du progrès scientifique et, deuxièmement, un refus d'assumer les acquis de la période turbulente, post-nietzschéenne, de la critique de la métaphysique. Pour faire bref, et sans dénaturer sa pensée, une nostalgie de la formation passée, d'abord historique, et une incapacité d'assumer la sécularisation.

Cette critique me semble très sérieuse et surtout bien ciblée. Car de quoi parlons-nous en ce moment ? D'une étape de l'évolution d'un système démocratique d'enseignement, système dans lequel la place spécifique de la philosophie a été défendue de manière constante depuis bientôt trente ans. Cette défense s'est effectuée précisément sur le fond de l'humanisme renaissant qui caractérisait le curriculum des collèges classiques, mais le temps est venu de mesurer sa rigueur et sa portée. Le système qui a suivi le régime des collèges classiques se caractérise par son universalité : tous les étudiants qui s'engagent dans des études collégiales, et ce devrait ètre idéalement le cas de toute la tranche d'àge, quel que soit leur profil d'orientation, se voient présenter une initiation à la philosophie étalée sur trois sessions du cours collégial. Cet objectif d'universalité, qui associe dans une même formation les futurs universitaires et les futurs techniciens. peut ètre critiqué, mais ce n'est pas mon propos aujourd'hui. Je l'aterprète d'emblée comme l'expression d'une volonté très affirmée de conserver à la formation générale une dimension philosophique universelle, c'est-à-dire valable pour tous dans la conduite de la vie, et de favoriser l'adoption d'un point de vue de réflexion sur les savoirs et sur l'existence. Pourquoi le même nombre de cours de philosophie pour tout le monde? Et pourquoi les mèmes cours pour tous, indépendamment des filières de formation? Ces questions se posent, mais elles ne modifient pas la place fondamentale de la philosophie dans la formation, place qui semble acquise et raisonnablement justifièe.

C'est précisément cette universalité qui entraine un questionnement nouveau sur les matières de l'enseignement: qu'est-ce qui, en effet, dans le regard porté sur l'avènement de la raison grecque, peut servir spécifiquement cet objectif d'universalité ? En quoi l'étude de la pensée grecque peut-elle contribuer de manière déterminante à la formation de l'étudiant contemporain?

On pourrait soutenir à bon droit que cette étude sert bien les objectifs de ceux qui se destinent à des études de lettres, d'humanités, ou encore dans les professions de la parole (droit, communications), une portion somme toute limitée du secteur pré-universitaire et située dans le droit fil de l'humanisme traditionnel, mais que pour tous les autres, une approche plus formelle, plus branchée sur la logique et l'épistémologie contemporaine ou, tout simplement, comme dans le troisième ensemble consacré à l'éthique, sur des problèmes liés à leur future occupation, serait plus fructueuse et bénéfique. 
C'est un argument très solide. On peut aussi argumenter, de manière très critique, que c'est rendre un mauvais service à la pensée grecque que d'y avoir recours de manière purement instrumentale, en faisant d'elle le cadre ou le terrain d'apprentissage d'habiletés formelles : n'y $\mathrm{a}-\mathrm{t}$-il pas une légèreté, voire une hypocrisie considérable à ne s'intéresser aux dialogues socratiques que pour en extraire les premiers rudiments de la méthode du débat rationnel et de l'elenchos? Les dialogues de Platon ne sont-ils là que pour nous instruire des formes primitives de l'argument, s'agit-il seulement d'un folklore, où les méchants sophistes sont vaincus par une métaphysique inspirée? Après tout, on assiste actuellement à une belle réhabilitation de la sophistique et des arts de la communication en Grèce, il suffirait de retourner à cela!Et puis, pourquoi ne pas aller puiser directement dans les formulations contemporaines de ces méthodes de raisonnement, de logique informelle? Qu'avons-nous besoin de faire semblant que la culture grecque ou même Socrate nous intéresse?

À ces questions, il n'y a plus de réponses toutes faites. Non seulement les questions commencent à peine de trouver de nouvelles formulations, mais les réponses héritées de la Renaissance manifestent plusieurs lacunes qui les rendent moins fécondes pour notre questionnement. Actualiser la pensée grecque, ce ne peut ètre répéter indéfiniment ce qui a légitimé sa fondation moderne dans l'Humanisme. Cette fondation, il faut le rappeler, cherchait fondamentalement à raccorder l'antique et le chrétien, en créant d'abord des modèles moraux. Le plus bel exemple de cette justification se trouve dans l'œuvre de Guillaume Budé $\tilde{e}^{1}$. Proche d'Érasme, avec qui il entretint une belle correspondance, Budé voulait soutenir que la connaissance de l'Antiquité classique était indispensable à l'étude de la théologie chrétienne. Pas seulement pour des raisons de pure philologie, comme l'accès à la langue grecque, mais pour des raisons de culture : la sagesse antique constitue, selon Budé, une véritable propédeutique morale à la pensée chrétienne qui en est comme l'achèvement historique. De Plutarque à Montaigne, le dessin de ce parcours ascétique était bien tracé. L'enseignement des belles-lettres et de la rhétorique était profondément inspiré par l'idéal de la perfection grecque, et c'est moins la philosophie que la littérature et l'histoire, porteuses de modèles héroïques, qui en étaient le centre. L'étude de la pédagogie des Jésuites, si profonde et si fondamentale, montre à cet égard une préoccupation de justification qui est sans pareille. Chaque morceau de l'étude du modèle antique doit se fonder sur des motifs rigoureux. On oublie, par ailleurs, que la Renaissance a d'abord été l'âge d'or de la scolastique, et non pas de la pensée grecque. On pourrait sans doute suggérer de retourner à cette scolastique formelle : n'ètait-elle pas la meilleure école d'argumentation?

Où trouver donc de nouvelles réponses? La situation actuelle se caractérise en effet, en Europe comme en Amérique, par une grande turbulence. Des philosophies curriculaires qui paraissaient inébranlables, comme le curriculum du gymnasium allemand ou du lycée

1. Principalement dans son ceuvre majeure, le De Transitu hellenismi ad christianismum Voir la traduction française, Le passage de l'hellénisme au christianisme, par Maurice Lebel, Sherbrooke, Éditions Paulines, 1973 (Centre d'études de la Renaissance de l'Universitè de Sherbrooke). 
français, ont été entièrement réformées ces dernières années et aucun de ces curriculums n'accorde une place universelle à la pensèe grecque, ni même à la philosophie. En Allemagne, compte tenu du primat historique de la philologie grecque depuis la réforme du gymnasium par Werner Jaeger et d'autres après la Première Guerre mondiale, primat demeuré incontesté jusqu'à récemment, le bouleversement actuel est une véritable révolution. En Angleterre, l'approche dite Greats, qui accorde une place significative à la culture grecque (surtout l'histoire et la politique, étudiées dans de grandes œuvres comme celles de Thucydide ou Platon), est de plus en plus réservée à une élite littéraire. En France, la réforme du baccalauréat a confirmé l'importance de la philosophie dans les filières d'élite, mais l'enseignement est essentiellement demeuré un éclectisme historique hérité du XIX ${ }^{e}$ siècle et orienté surtout vers l'apprentissage de l'art de la dissertation. Laccent sur la philosophie des sciences dans la filière scientifique s'est par ailleurs renforcé au détriment de l'histoire de la philosophie. Aux Etats-Unis enfin, la tendance très nette va vers une spécialisation des premiers diplōmes post-secondaires, et seule une minorité de collèges d'élite, influencés par des objectifs d'arts libéraux, et en particulier par la tradition des Great Books de Chicago, maintient un objectif d'initiation à la philosophie dans laquelle la pensée grecque a encore sa place. La grande majorité des collèges (junior colleges) présentent un entraînement de informal logic, de critical thinking, et parfois un cours très gènéral de basic issues. On y a par ailleurs conservé les communication arts, qui sont l'équivalent de l'ancienne rhètorique et qui indiquent l'importance toujours actuelle de la parole publique pour l'élite. Il y a donc actuellement dans l'enseignement post-secondaire un net recul des humanités grecques et un repli manifeste vers les formations d'élite. Ce constat peut nous attrister et il ne décrit sans doute pas une situation définitive, l'état actuel de la réflexion curriculaire pourrait se renverser. On note, par exemple, dans certaines écoles secondaires et collèges d'élite un retour aux langues anciennes, et dans quelques universités du top ten américain, on fait la promotion de programmes collaboratifs de prestige philosophie-philologie grecque.

Mais les faits sont là et on doit se demander : quel est le sens de notre propre retour à la pensée grecque ? Je reviens à la question de mon ami Vacher : le retour aux Grecs est-il réactionnaire ? L'Antiquité peut-elle ètre moderne et servir le projet critique d'un curriculum universel et démocratique? Pour structurer la discussion, je voudrais repasser par les deux critiques que j'ai rappelées à l'instant. La première a trait à l'urgence et à la complexité d'une nouvelle fondation de la rationalité. Cette critique entraine la formulation d'un argument historique essentiel. On peut, je pense, soutenir que le retour à l'émergence de cette rationalitê, à son moment originel et à sa posture philosophique propre, est le moyen le plus puissant pour en montrer la nécessité et la singularité. J'insiste sur ces deux aspects : d'abord la nécessité de l'adoption du point de vue rationnel dans le regard sur l'existence, point de vue qui a résulté d'une décision historique singulière, dont notre culture est l'héritière. Nécessité de la raison, mais également singularité de l'expérience historique de la raison : il s'agit de deux aspects différents, mais indissociablement liés, de la 
culture grecque. Ce premier argument a été reformulé magistralement par Husserl et cette formulation dépasse de beaucoup les formulations de la Renaissance. J'y consacrerai le premier moment de mon exposé.

La deuxième critique suscite un argument plus difficile à énoncer, mais je vais m'y risquer. Contre le reproche de crainte de la sécularisation, on peut en effet soutenir que la pensée grecque est précisément une pensée séculière et cela, en dépit de ses composantes mythiques manifestes, même au cœur de la métaphysique, et qu'y retouner est le meilleur moyen de saisir la force de ce qui l'a occultée, en la captant entièrement - la métaphysique chrétienne -, de manière à saisir les enjeux de toute pensée aujourd'hui, en particulier son affrontement universel avec de nouveaux mythes ou de nouveaux fondamentalismes et la critique, plus nécessaire que jamais, du scientisme, c'est-à-dire de la religion de la science. Le retour aux Grecs n'est donc pas le repli métaphysique qu'on pourrait croire et il y a des arguments contemporains, différents des arguments chrétiens de la Renaissance, pour l'appuyer.

Ces deux arguments ne viennent peut-être pas à bout d'une position centrée uniquement sur le privilège formel de la logique ou de la discussion de la scientificitè, au détriment de toute historicité, mais ils ont l'avantage d'ancrer l'affirmation de la rationalité dans l'histoire de la culture européenne dont nous sommes les héritiers. Cet ancrage est le point limite de notre réflexion, le moment où toute discussion sur les origines et les finalitès d'une formation devient une réflexion sur l'identité : le privilège de la rationalité est-il un privilège neutre, coupé de l'histoire de la pensée occidentale, ou n'est-il pas plutôt le résultat le plus fécond, le point d'aboutissement de cette histoire? En répondant à cette question, nous serons au vif de la question de l'actualité de la pensée grecque, de son actuelle fécondité, de sa véritable modernité. Nous serons également mieux en mesure d'élaborer des stratégies de lecture et de formation qui découleront de ce privilège de la source grecque.

Dans ses conférences sur la crise de l'humanité européenne, Husserl a formulé pour nous de manière radicale le problème de cette translatio studii qui demeure le problème de la paideia moderne. Quelles études devons-nous transmettre? En quoi contribuent-elles à la formation moderne et en quoi présentent-elles une aptitude à penser le présent? Sa question, dont la formulation si riche implique aussi bien la raison que l'histoire, peut nous servir de point de départ: qu'est-ce que la culture dont nous héritons quand nous disons que cette culture se donne pour fondement la raison? La réponse de Husserl est connue : pour lui, c'est la figure spirituelle de l'Europe qui, dans son concept, englobe toutes les cultures de la raison, y compris l'Amérique. "Il s'agit, écrit-il, de l'unité d'une vie, d'une activité, d'une création spirituelles, avec tous les buts, tous les intérêts, soucis et peines, avec les formations téléologiques, les 
institutions, les organisations ${ }^{2}$ ". Les aspects hégéliens de cette réponse n'échapperont à personne - je pense à l'esprit, à son développement dans une histoire orientée, à sa récapitulation dans une culture -, mais laissons cet optimisme de côté. Concentronsnous sur l'essence de cette activité : cette figure spirituelle de la rationalité européenne demeurerait ininterprétable si on ne la ramenait à son histoire qui, selon Husserl, est l'idée philosophiquement immanente de l'histoire de l'Europe. Je voudrais citer la suite de ce passage important :

[...] du point de vue de l'histoire universelle, [cette idée] se fait connaitre comme l'irruption et le début du développement d'une nouvelle époque de l'humanité, l'époque de l'humanité comme telle, qui désormais ne veut et ne peut vivre que dans la libre formation de son existence, de sa vie historique, par l'idée de la raison, par des tâches infinies.

L'idée de cette universalité était chère à Husserl, et nul ne sait comment il voudrait la défendre aujourd'hui dans un monde éclaté où l'Europe historique a traversé et traverse encore des périodes de ténèbres et de désintégration spirituelle désespérée. Husserl n'a pas connu la période hégémonique du stalinisme, ni l'affrontement de l'Occident avec un Orient musulman en proie aux confusions de l'intégrisme, affrontement qui semble actuellement vouloir forger la structure émergente des limites européennes. Quand il prononce ces conférences à Vienne et à Prague en 1934 et 1935, il a néanmoins devant lui la figure très nette du fascisme. Pour lui, cet idéal universel se développait d'abord dans l'espace historique de l'Europe, dont le lieu de naissance, à la fois emblème et essence de la rationalité, pouvait peut-être constituer un salut devant le fascisme. La dimension de la raison qui le sollicite alors, comme ce sera également le cas pour Heidegger, est son caractère époqual, qui évoque à la fois la précarité et l'unicité de l'expérience historique. Que l'histoire l'ait amèrement trompé n'entame pas son argument.

J'attire votre attention maintenant sur ce lieu de naissance, sur le fait que la Grèce, dans le texte de la Krisis, joue un rôle essentiel. Nous devons essayer de le comprendre, dans la mesure où il y va de notre propre héritage, de ce qui, dans cet héritage, a trait à notre époque et de la question même que nous posons. La Grèce n'est pas une forme historique arbitraire, une incarnation parmi d'autres d'une idéalité, à laquelle on pourrait juxtaposer par succession d'autres exemples d'humanité : en tant qu'origine emblématique de l'Europe, elle constitue le telos universel de l'humanité. Sur ce plan, Husserl ne fait intervenir aucun concept d'Occident, ou de culture occidentale, qui limiterait dans sa pensée la portée du telos européen. Il n'y a pas non plus pour lui d'Orient de la raison, comme cela existe de manière si fascinante pour nous désormais. Pour tout dire, la raison

2. E. Husserl, La crise des sciences européennes et la phénoménologie transcendantale, trad. fr. de Gérard Granel, Paris, Gallimard, 1976, p. 352. Le texte que je place au centre de mon exposé est celui de la conférence de Vienne (7 et 10 mai 1935), donnée sous le titre La crise de l'humanité ewropéenne et la philosophie. Dans un ouvrage récent (Giuseppe Cambiano, Le retour des anciens, Paris, Belin, 1994), on pourra trouver une analyse de plusieurs approches de la pensée grecque dans l'histoire contemporaine de la philosophie, notamment chez Heidegger et Gadamer. 
ne rencontre chez lui aucune altérité, position en soi excessive, téméraire. Tout ce qui est étranger à l'Europe, parce qu'étranger à la vie de la raison (Husserl cite l'exemple de l'Inde), vient-il en contact avec l'Europe qu'il en subit aussitôt le telos déterminant, et cette essence est d'abord et d'emblée grecque. La Grèce est donc l'essence historique de l'expérience européenne.

La témérité, et en un sens la naïveté, de la proposition ne doit pas nous détourner de son sens. Elle ne se fonde pas en effet sur ce qu'on pourrait appeler une nécessité de type hégélien. Husserl était au contraire sensible à la possibilité que le développement de la raison soit interrompu, brisé par le fascisme. L'Universalité du destin de la raison ne contredit donc pas sa précarité historique. On aurait tort, par ailleurs, de limiter la prétention de ce rationalisme, exacerbé par la menace de l'irrationnel fasciste, à la mise en jeu d'une rationalité purement scientifique, comme si cette rationalité ne déterminait pas d'emblée un humanisme et une éthique. Je voudrais ici faire le raccord avec ma première question. Ce n'est pas, en effet, dans son lien purement spéculatif ou dialectique à l'essence du réel que le monde grec s'impose à nous comme monde de la rationalité européenne, mais en tant que ce monde déploie pour s'y engager le modèle d'une humanité normative transcendante. Il s'agit d'un modèle de vie. Le terme mème de philosophie a été forgé pour nommer cet idéal d'une recherche de la sagesse qui ne soit pas purement spéculative, en grec théorétique; la philosophia recoupe, en effet, tout ensemble et d'une mème jetée le projet physique qui, d'Anaximandre à Aristote, entreprend de saisir la physis comme monde soumis à des lois et susceptible de manifester sa nécessité, mais également le projet métaphysique qui dispose ce monde dans une histoire spirituelle qui la fait transiter du mythe orphique de l'âme à la théologie chrétienne de la rédemption.

Husserl ne considérait pas de première importance les questions éthiques ou théologiques, tant la puissance du projet rationnel lui semblait garante de l'établissement rationnel de la culture. Son optimisme, qui sera repris par Cassirer, était en fait le résultat d'une confiance et il n'est pas certain que nous soyons encore capables de la partager entièrement, sinon comme le projet ou le défi de la raison. Fondée sur la raison, une culture ne pourrait que déployer sa bonté spirituelle et manifester son essence humaine positive. Avant d'ètre le protreptique à la vie bonne et sage qu'elle avait été dans l'Antiquité, la philosophie était définie par Husserl, dans son essence universellement grecque, comme " science du tout du monde, de l'uni-totalité de tout l'étant ". Ce regard sur la Grèce est le regard d'un rationaliste, et non d'un moraliste comme Pierre Hadot par exemple ${ }^{3}$, mais dialectique et protreptique sont des démarches indissociables : changer la vie et chercher le vrai sont les deux faces du toumant grec. L'une est socratique, l'autre est aristotélicienne.

Dans sa double vocation, ce projet en lui-meme constituait une détermination de l'humanité en tant que porteuse d'idées, de pensée. Pour Husserl, la philosophie dans sa source grecque a fait subir à

3. Notamment dans son récent Qu'est-ce que la philosophie antique?. Paris, Gallimard, 1996. 
l'idée d'humanité une transformation définitive : avec la philosophie grecque, l'expérience humaine est d'abord et avant tout l'expérience de la pensée et des idées. La dialectique socratique manifeste, dès l'origine, comment cette expérience se déroule de façon communicative, organique et politique, comment elle "éveille un nouveau style de l'expérience personnelle dans son champ vital" ". Elle dresse la force de l'argument et de la conversation contre le pouvoir de l'autorité et de la rhétorique, ce qui constitue son geste premier. Mais elle le complète aussitôt d'un geste qui approfondit le premier : elle identifie la conscience, en la créant, contre le règne de l'extériorité. recueillant en quelque sorte dans sa parole la force intérieure d'Antigone.

Profondément enraciné dans le devenir infini de la vie éthique, l'idéal grec de cette conscience argumentée, du souci de l'âme, de la melete thanatou, de la formulation du bien vivre, dont l'exemple est d'abord celui de Socrate dans le Criton, est à la fois absolument irréductible au projet de l'étude de la physis et son corollaire le plus naturel, le plus existentiel. Là se trouve l'essence de la sagesse grecque, cette confiance dans la double puissance de la raison: définir l'humanité comme humanité sage et poser le monde comme nécessitè. Même si Husserl ne prend pas le soin de définir ce qu'il entend par spirituel, même si la religion ne joue aucun rôle dans la formulation de l'idéal de l'âme, on comprend que la définition de la philosophie par la spiritualité implique une sédimentation de l'entreprise rationnelle. Il y a, en effet, plusieurs niveaux de vie, et chacun est convoqué dans l'apprentissage de la philosophie. De la même manière, l'enseignement de ce geste inaugural est d'abord une invitation à le répéter pour soi-même : trouver sa conscience, l'appuyer sur une parole nècessaire, articuler cette parole sur une requête de vérité.

L'exercice de la philosophie, en dépit de cette sédimentation, demeure cependant unique et son déploiement historique, même s'il présente une diversification des projets épistemiques et spirituels immaitrisable, ne risque jamais de perdre cette unité, en raison précisément de son rattachement à son origine grecque comme décision de penser. Contrairement à Heidegger, dont les approches sur la question de lhistoire de la philosophie sont si paradoxales, ce qui l'amène à une fracture radicale de l'expérience grecque de la pensée en deux moments antithétiques - les présocratiques de la présence et le Platon de l'absence - Husserl se reconnait engagé dans un procès unique, et infini, de réalisation de la tâche spirituelle de la philosophie. Là où, pour Heidegger, la Grèce a constitué une forme de trahison originelle, conduisant à ce premier nihilisme qu'il a dénoncé dans l'entreprise de Platon, Husserl parle d'une mission historique, d'une perpétuelle mutation dans le sillage grec. Selon sa formule radicale, la Grèce entraine une historicité nouvelle. Dans ce modèle, il faut insister, largument socratique et la science aristotélicienne appartiennent au mème monde, au même geste, à la même décision qui est l'essence de la philosophie.

4. E. Husserl, La crise des sciences européennes et la phénoménologie transcendantale, p. 356. 
C'est à saisir cette historicitè, à la transmettre comme fait déterminant de notre situation spirituelle, que l'enseignement de la pensée grecque est principalement adonné. Si la philosophie mérite cette place privilégiée dans l'édifice complexe des sciences contemporaines, c'est précisément parce qu'elle appartient à la fondation mème du regard scientifique sur l'ètant. Comme cette fondation est grecque par essence, nous pouvons dire que nous sommes essentiellement grecs d'emblée. Le cœur de la translatio studii contemporaine, sa ligne de force déterminante, est donc l'expérience grecque assumée dans tout l'héritage européen d'une posture rationnelle devant la totalité de l'expérience humaine, toujours à la fois historique et physique, spirituelle et scientifique. Briser cette translatio, abandonner cet enseignement au profit de techniques banales, toujours susceptibles d'être asservies à la déraison, c'est prendre le risque de fracturer un héritage qui non seulement a produit la science et la critique, mais a rendu nécessaire la posture qui l'engendre.

Cet argument repose sur un lien plus étroit qu'on ne le conçoit d'ordinaire entre la philosophie et les sciences. La position habituelle qui consiste à placer la philosophie en position critique et dominante par rapport aux sciences n'a rien de grec: la pensée grecque juge la doxa et le mythe, mais elle estime et place au-dessus de tout la pensée du nécessaire, dans quoi elle identifie la science et la philosophie. L'argument philosophique, qu'il s'agisse de l'enchaînement formel qui produit la proposition scientifique, qu'il s'agisse du raisonnement dialectique, appartient toujours au même corps de méthode. Cette méthode est continue, elle est cohérente. On voit bien la jointure avec Husserl : la philosophie, dans la conception husserlienne, comprend les sciences, elle les implique toutes potentiellement et le geste mème de la science est impensable sans la décision philosophique qui l'inaugure et l'accompagne, le produit de la science est impensable sans le cadre philosophique qui le met à l'épreuve et le critique; la méthode de la science ne peut ètre énoncée sans la contribution de la logique.

De ces trois dimensions - décision, critique, méthode - , Husserl a voulu prédiquer l'infinité et l'ouverture de cet horizon infini est, depuis la pensée grecque, la perspective mème de toute pensée. Et il a proposé de faire de cette infinité l'horizon spécifique de la culture universelle, relayée de la Grèce en Europe. Accepter cet idéal et cette tàche, c'est d'abord refuser de construire dans une opposition stérile une soi-disant modernité scientifique et la présentation du monde grec comme topos archaïque du rationnel : le monde grec a ouvert définitivement, d'une manière qui ne s'est pas produite en Orient ou en Israël ou même dans le monde musulman, un horizon infini de rationalité. L'Islam a cherché pendant une période qui fut grande à recueillir et tranformer la pensée grecque, mais ses maitres coraniques ont avec le temps abandonné cet héritage. La philosophie, dans sa double dimension de sagesse et de science, est la culture de l'Europe et son essence, inaliènable, est l'expérience grecque.

Avec ses limites, certes, mais aussi sa grandeur. Ce point me semble essentiel et je voudrais m'y attarder un instant. S'il est certain que la philosophie du $X^{e}$ siècle peut ètre présentée comme une critique du rationalisme, comme un énoncé de ses limites - il suffira 
d'évoquer la critique de Nietzsche, la pensée extatique de Heidegger et son relais chez Derrida et Rorty - je pense que l'idéal husserlien d'un horizon infini peut être retrouvé et maintenu en deçà de l'entreprise déconstructive. La décision pour la raison ne conduit pas, ne doit pas conduire à l'exigence infantile d'un ordre transparent et d'une rationalité sans limites, exigence qui se retourne ensuite en plainte mélancolique au constat de l'absence de cette rationalité idéalisée. Dans ses entretiens publiés sous le titre Autonomie et solidarité, Habermas fait remarquer à quel point nous avons du mal à accepter une perspective faillible et une rationalité limitée, en raison de nos exigences idéalistes ${ }^{5}$. Nous préférons tout refuser plutôt que de nous résigner à une rationalité partielle, fragile. Cette critique, qui vise maladroitement la pensée de Derrida, atteint de manière oblique Husserl.

L'idée husserlienne d'une tâche infinie de la rationalité, malgré son optimisme exagéré, paraìt à cet égard plus proche de l'intuition grecque d'une raison contingente, finie, mais paradoxalement ouverte, et pour ainsi dire happée, par l'appel d'une compréhension infinie. Pour le faire voir, on peut faire valoir deux arguments. Premièrement, la position de Husserl semble fidèle à ce qui, dans le projet grec, s'appelle theoria, dans son opposition à la praxis et à la poiesis. Le privilège constant de la theoria n'a jamais signifié que l'accès à la theoria était stable, définitif ou mème achevé. Seuls les objets de la theoria, chez Platon comme chez Aristote, sont nécessaires : ce sont les Formes éternelles, les mouvements éternels ; mais en tant que nécessaires, ces objets exigent précisément la tâche infinie de la philosophie. Les limites de la raison humaine lui imposent des démarches contournées, des reprises, des recours au mythe, des procédures analogiques, des dialectiques qui exigent du temps et qui ne sont pas compatibles avec l'idéal d'une contemplation continue ou d'une extase. Il y a donc un infini grec de la tâche philosophique qui peut nous réconcilier avec sa finitude, son historicité, sa temporalité et le caractère vulnérable, toujours fragile, de ses constructions. Cet infini représente un travail, une responsabilité, une tàche et en ce sens, il s'impose au point de départ de la formation. Cet infini est par ailleurs mesurable à une certaine déconstructibilité de la pensée grecque. Toutes les idées sont infinies - vérité, valeur, bien authentique, forme de vie, liberté - et tous ces infinis sont ancrés dans l'expérience finie de quelques penseurs grecs. Dans ce rapport fini/infini, où chacun est libre de lire tellement d'arbitraire (qu'est-ce qui m'a amené moi, à lire et à saisir ceci plutōt que cela ?), s'exerce un apprentissage de la modestie (rappelons-le, l'hubris était le péché cardinal de l'esprit grec) et une initiation à la solidarité de la communauté de recherche et de dialogue : ce sont les idées que Rorty a exprimées dans le beau langage des tâches de l'édification ${ }^{6}$.

À cette première raison de considérer l'importance de l'idée husserlienne de l'infini des tâches de la raison comme essence grecque s'ajoute la dimension politique de sa détermination. Cet

5. J. Habermas, Autonomy and Solidarity, revised edition, edited by Peter Drews, London, Verso, 1992 (première édition : 1988), p. 198 ss.

6. R. Rorty, L'homme spéculaire, Trad. fr. de T. Marchaisse, Paris, éditions du Seuil, 1990, p. 393-410. 
infini, dès les premiers penseurs, nous comprenait nous-mêmes comme partie prenante d'une communauté spirituelle destinée à le recueillir. Je ne pense pas seulement au monde de l'hellènisme, relayé par la culture romaine, par Byzance et par la Renaissance italienne. Je pense à la totalité des moments cruciaux de la translatio studii. moments dans lesquels la culture européenne s'est interrogée sur la responsabilité de la transmission de l'idéal grec de la culture. Il y a des moments plus nets que d'autres, la pédagogie des Jésuites par exemple ou le romantisme allemand. Mais dans chacun de ces moments, c'est l'idée husserlienne de l'infinité des générations de la rationalité, comprise politiquement comme communauté de l'amour des idées infinies, qui a été le moteur de l'évolution ${ }^{7}$. Il n'y a jamais eu d'abandon, de renoncement, de retour à une forme archaique qui serait l'équivalent d'une pre-theoria. C'est pourquoi la critique de Heidegger et son relais chez Derrida et Rorty sont si importantes pour nous : elles mettent en jeu les conditions mèmes de notre réponse à une nouvelle translatio studii, elles imposent un regard sur la raison qui doit tenir compte des faillites de notre époque, des dangers, des risques. Ces critiques désignent un lieu vide qui doit toujours être réoccupé par le travail de la raison. Abandonné, il se désagrège. Investi, il ne cesse de se recomposer.

Dans la contestation actuelle des privilèges de la philosophie, plusieurs critiques américains ont reproduit le débat de l'idéalisme allemand relatif à la littérature : ne faudrait-il pas confier à la littérature l'essentiel de cette mission de formation, compte tenu du fait que la philosophie a démissionné de sa vocation de paideia universelle, qu'elle n'enseigne que la logique, qu'elle n'a plus rien à dire sur l'historicité et la contingence de l'expérience humaine et qu'elle a renoncé au discours de sagesse ? C'est l'argument de Rorty, un argument à la fois surprenant et un peu usé. Il s'agit cependant d'une position profondément non grecque dans son geste de retoumer au privilège de la représentation sur l'argument. La position de Derrida, à la fois plus inquiète et plus œcuménique, me semble un meilleur recours ${ }^{8}$. Husserl, bien entendu, n'aurait jamais considéré pareil retournement, même en acceptant toutes les limitations de l'expérience historique de la rationalité. Pour lui, la theoria grecque est d'abord une attitude, une décision qui affecte tout le mode d'existence, quels que soient les succès et les échecs dont elle sera suivie. "C'est dans la permanence de ce style, en tant que forme normative, que se déroule la vie comme vie chaque fois déterminée ${ }^{9}$."

Guelle est notre position sur cette question? Nous pourrions en dèbattre, mais en l'évoquant nous voyons que toujours déjà ce débat appartient à la philosophie dans son moment grec. L'attitude purement théorétique, qui aboutit à substituer la philosophie à la forme de la littérature, et notamment de la tragédie, dans les privilèges du

7. E. Husserl, La crise des sciences européennes et la phénoménologie transcendantale, p. 359.

8. Je pense ici en particulier aux réflexions européennes de Derrida, à partir d'un texte de Paul Valéry, dans L'autre cap, Paris, éditions de Minuit, 1991. Notamment, p. 33 ss.

9. E. Husserl. La crise des sciences européennes et la phénoménologie transcendantale, p. 360. 
savoir de la culture, a souvent été mise à l'épreuve dans l'histoire de la culture européenne. Ce fut le cas de manière forte dans la contestation du romantisme allemand. Une réponse possible est la réponse politique : la theoria grecque ne vaut pas seulement par elle-mēme, elle vaut par ce qu'elle entraine avec elle. Husserl est à cet égard, une fois de plus, l'héritier de Hegel quand il insiste sur le caractère central de la theoria dans la production de la communauté. L'amour ici joue un rōle vital, organique : la Grèce apporte l'exemple d'une culture où la communauté dépasse sa violence intrinsèque par la position normative d'un idéal d'éveil et de discussion, dont le plus bel énoncé est l'oraison funèbre de Périclès dans le texte de Thucydide, ou encore le Phédon de Platon. Ces éloges de la philosophie, et de sa supériorité par rapport à la littérature, sont en fait des éloges politiques de la philosophie. La dimension politique de la raison grecque est donc inséparable de sa dimension purement théorétique, autant que celle-ci l'est de son déploiement éthique. Il n'est cependant aucunement nécessaire d'adopter cette posture politique, qui remonte à l'idéal hégélien d'une communauté rationnelle, pour penser dans le moment grec la conjonction de la littérature et de la philosophie.

Renoncer à ce primat de l'expérience grecque, ce serait donc - il faut insister à l'encontre de ceux qui croient utile de le revendiquer se priver non seulement du moment de fondation de la rationalité, ce qui est la formulation habituelle de la nécessité de retourner aux Grecs, mais de ce qui dans notre situation me semble le plus précieux : leur essentielle universalité. Dégager la philosophie de cette attitude ouverte sur l'infini de l'histoire, désimpliquer le projet physique de l'éthique et du politique comme si cela était pensable ou possible, c'est renoncer à l'essence mème de la philosophie dans sa décision de rationalité. La conséquence me semble immédiate et risquée : sans cet ancrage dans l'universel grec, la rationalité devient une voie parmi d'autres, un chemin contingent de l'histoire et l'Europe elle-même une expérience condamnée et abandonnée aux sous-produits technocratiques de la raison. S'il me semble utile de revenir sur la position de Husserl, c'est qu'elle accorde à l'idéalité de la pensée grecque, à son ouverture infinie, une importance mesurée au développement de la rationalité dans l'histoire. Il n'y a, en effet, aucune période de l'histoire européenne qui ait été capable de réarticuler son idéal de raison sans en retrouver le fondement dans la communauté de la theoria grecque : nous connaissons bien les deux moments les plus grands de cette histoire, la Renaissance et les Lumières. L'un et l'autre ont mis au centre de leur pensée la reformulation de la translatio studii: comment actualiser la pensée grecque, comment retrouver l'énergie intellectuelle de la culture grecque, comment restimuler sa vitalité démocratique? Plusieurs textes pourraient ici nous servir de guides, j'ai cité la translatio de Guillaume Budé, je pense également à Kant et à Hegel. Il faudrait y revenir.

Je conclus ce premier morceau de mon exposé en insistant donc sur le fait que le retour à la rationalité grecque n'est pas un repli superficiel sur une tradition rassurante. C'est un geste courageux, dans lequel nous cherchons à nous réapproprier l'attitude fondatrice de notre culture, de manière à la conserver comme fondement de 
notre identité. Les formulations de Husserl nous paraissent-elles démesurées qu'elles n'en constituent pas moins le projet infini d'une culture à faire, d'une humanité à construire. Cet universel nous semble-t-il devoir être déconstruit qu'il ne l'est que par les instruments mêmes qu'il rend disponibles pour notre critique.

\section{II}

J'en viens maintenant à la deuxième perspective que j'ai évoquée au début. Le retour aux Grecs constitue-t-il une sorte de repli sur une attitude métaphysique, reflétant la crainte d'une société sans bases et l'incapacité de s'ajuster à une modernité post-chrétienne? Nous devrons ici tenter de mieux saisir ce qu'il en a été du développement de la métaphysique et prendre au sérieux les critiques contemporaines de l'onto-théologie. Il me semble indubitable, malgré plusieurs beaux travaux récents allant en sens contraire, que la pensée grecque de Platon, d'Aristote, de Plotin a clairement construit cette identification de l'être et de la divinité, dans un respect fondamental à l'égard de son origine homérique et de la théologie des immortels. Si donc nous retournons à la pensée grecque, nous sommes inéluctablement mis en présence d'un monde dans lequel le projet de saisie rationnelle de la physis trouve sa limite dans l'affirmation d'un au-delà. Cela fait-il de notre retour aux Grecs un retour vers l'onto-théologie en tant que telle? Que désirons-nous montrer ? Quel est le rapport entre cette métaphysique et le mythe?

Je commencerai par un point précis. Je suis saisi de l'importance, parmi les textes qui sont placés au centre de la pédagogie de ce premier cours de philosophie au collégial, de l'allégorie de la caverne de Platon. Ce texte occupe certes un rōle central dans toute la tradition de la métaphysique, on y a toujours retrouvé le condensé de la doctrine du dualisme : dualisme logique, dualisme épistémologique, dualisme moral. Mais il y a un risque considérable à le présenter uniquement comme le texte fondateur des deux mondes. On oublie en effet, quand on lit ce texte, de signaler le fait que le mythe qui en commande l'interprétation en fait intégralement partie, même s'il n'est pas présenté avec l'allégorie dans le texte de la République. Comment comprendre en effet la situation existentielle de ces prisonniers, dans lesquels Platon nous invite à reconnaitre notre condition mortelle, sans poser les deux questions suivantes : qui est à l'origine de cette situation misérable d'aveuglement et de finitude ? Et comment se fait-il que certains prisonniers accèdent à la libération par la philosophie? Platon n'a donné aucun élément spéculatif pour répondre à ces questions, et celui qui cherche une réponse doit la trouver dans le mythe orphique qui, en lui fournissant sa doctrine de l'âme, commande tout le platonisme. Ce mythe est un mythe de chute et de résurrection, dont un des épisodes les plus connus a transité dans la doctrine de la réminiscence et de la métempsychose.

Pourquoi revenir sur ce point? Tous les étudiants qui lisent ce texte pour la première fois sont intéressés par le savoir spécial du philosophe, et ils comprennent d'emblée cette situation d'emprisonnement dans l'opinion et dans la représentation. Mais ils ne songent pas que la question du mythe doit également les solliciter. Ce mythe de l'âme, comme d'autres, fait partie intégrante de la pensée grecque, 
dont il manifeste les limitations. L'onto-théologie elle-même appartient d'emblee au mythe orphique, elle en est pour ainsi dire le développement spéculatif. Même des penseurs fascinés par le rationalisme platonicien, comme Plotin, ont été incapables de se priver d'y avoir recours. Cette prégnance du mythe - qu'il s'agisse du Phèdre ou de la République -, ce rapport complexe de la croyance et de la pensée rationnelle étaient néanmoins, dans le monde grec, dans une position d'équilibre. Pour le comprendre, il faut voir comment cet équilibre a été renversé quand le mythe orphique a été entièrement intégré par la théologie chrétienne. Dans cet âge médiéval, qui va d'Augustin à Nicolas de Cues, le développement d'une sagesse de la foi a mis sous tension, de manière souvent très paradoxale, le projet rationnel des Grecs. Ce projet a été assujetti à la théologie, la métaphysique est devenue plus essentielle que la physique, ce qui aurait scandalisé Aristote et sans doute aussi Platon.

La critique de la raison, et a fortiori son assujettissement au mythe, en fait n'a jamais èté une attitude grecque. Même en en reconnaissant les limites et en cherchant à la protéger de l'hubris technique, mème en insistant sur le caractère infini de sa tâche, les penseurs grecs n'ont jamais cessè de faire l'éloge du pouvoir de la sophia. Leur pensée était une pensèe du pouvoir de la raison, par rapport à laquelle le mythe n'était toujours qu'une forme de concession, de résignation. L'entreprise aristotélicienne montre à quel point la philosophie fut rapidement travaillée de l'intérieur par le désir d'étendre le plus loin possible l'enquète de la physique et de la philosophie première. La grandeur du projet rationnel était justement de se projeter le plus loin possible, le plus haut possible. Ce réflexe de critique des prétentions de la raison fut plutôt celui de la pensée chrétienne, dans un contexte de turbulence gnostique où la raison paraissait emportée par une sorte de délire et où les sagesses antiques ne semblaient montrer que des défauts ou présenter des exigences impossibles. La foi parut alors plus raisonnable qu'une pensée entrainée par l'hystérie des voyages astraux, des drames de chute, etc. et plus accessible, plus universelle que la sagesse. En croyant reconnaitre dans toutes ces péripéties les positions fondamentales du platonisme, le christianisme ne faisait que se l'approprier. Rien ne justifie dans le platonisme une connaissance supérieure à l'exercice de la raison. Le reste est amour, comme Plotin et Proclus l'affirmeront clairement.

Le retour aux Grecs constitue-t-il une sorte de fuite dans laquelle on pourrait lire un refus de la sécularisation ? Cette manière de voir supposerait que la vision grecque du monde est celle qui a été tout entière rèinvestie dans la conception chrétienne, à la fois en tant que metaphysique fondatrice et mythe orphique de la religion de salut qui a donné à l'Occident sa forme spirituelle la plus profonde. Retourner aux Grecs, dans cette perspective, ce serait chercher à retourner à une origine ou à une essence du christianisme, comme on chercha à le faire à la Renaissance. Par exemple, encore une fois, chez Guillaume Budé. Il s'agit, me semble-t-il, précisément de faire autre chose, c'est-à-dire de montrer que le christianisme, dans sa force théologique autant que dans son effort métaphysique, n'a jamais été qu'une modalité, contingente et historique, de transmettre et 
d'interpréter l'héritage grec, et non pas son développement essentiel. Il y aurait beaucoup à dire ici sur la comparaison nécessaire de cette interprétation (Plato naturaliter christianus) avec la philosophie des Arabes, d'emblée centrée sur le projet de la physique. C'est en effet cette philosophie qui a été à l'origine du renouveau aristotélicien du $\mathrm{XIII}^{e}$ siècle. Mais n'oublions pas que le christianisme, avant d'être aristotélicien, a d'abord été profondément néoplatonicien, c'est-à-dire essentiellement préoccupé de sa fondation théologique dans la pensée grecque ${ }^{10}$.

Le christianisme a été, il est vrai, une continuation de la métaphysique grecque, il a cherché à en assumer délibérément l'héritage. mais il en a été aussi et surtout le renversement et l'intérêt d'un retour à la pensée grecque est précisément de rendre possible l'accès à un monde pré-chrétien qui montre à la fois les richesses de l'un et de l'autre. Qui montre notamment la position de pensée d'une culture qui place la raison devant ses dieux, qui construit la démocratie et le débat public sur l'espace même de ses autels. Je voudrais m'arrêter un instant sur ce point et tenter de montrer que l'intérêt d'un retour à la pensée grecque dans une formation de philosophie se fonde très précisément sur cet équilibre pré-chrétien de la raison et du mythe, dont la marque essentielle est l'absence de la composante de la foi et l'importance de la critique des représentations doxiques et mythiques du monde.

Dans la situation contemporaine d'une résurgence de mythes fondateurs de toutes sortes et d'un retour du religieux, cet équilibre ancien met en perspective la fragilité de l'équilibre actuel : la comparaison met en relief la force d'une position dans laquelle la raison ne renonce à ses prétentions que dans les lieux les plus ultimes de son questionnement, et non pas dans le domaine même de la theoria. Nous savons comment la période de l'hellénisme tardif a été envahie par toutes les formes de la gnose, de l'hermétisme, des savoirs occultes. Qu'en dépit de cette phase très turbulente, et en dépit également des transformations de la théologie chrétienne avant le XIII siècle, le rationalisme ait été capable de se maintenir comme idéal de la culture et comme position existentielle mérite d'être expliqué. Cette persistance n'est en fait compréhensible que dans le maintien systématique, à la fois justifié et élaboré en stratégies, de la paideia philosophique grecque, c'est-à-dire d'une éducation centrée sur la theoria. La culture médiévale a profondément modifié cet équilibre en conférant à la théologie et à la foi une priorité qui devint l'équivalent d'une domination de la raison. Ce nouvel équilibre n'avait plus rien de grec, même si en sous-œuvre la physique et la dialectique conservaient leurs privilèges.

Je donnerai un exemple, repris d'une époque de transition antérieure. La culture romaine, dans un premier moment païen, mais ensuite également chrétien, aurait parfaitement pu se constituer comme une culture purement religieuse et juridique, et interrompre la

10. Sur plusieurs de ces questions de la transmission et leur impact sur la définition de l'Europe, on peut lire l'essai de Rémi Brague (Europe, la voie romaine, Paris, Critérion, 1992) qui tente dans une perspective chrétienne la réhabilitation d'une certaine * romanité *. 
continuité de la curiosité et de la rationalité grecques. Nous savons tous combien elle est pauvre philosophiquement, comment elle s'est replièe sur une théologie spéculative très èlaborée, vénérée par des philosophes comme Cicéron ou Lucrèce, et comment elle a investi la formulation des lois et les règles de la rhètorique, comme si rien de la physique d'Aristote n'avait d'importance, comme si rien de la métaphysique n'avait encore de sens. Le paradoxe est donc pour nous de constater qu'en dépit de cela, la culture romaine a maintenu pour ses classes d'élite une éducation grecque, c'est-à-dire une éducation à la philosophie, présentée comme le couronnement des arts libéraux. L'édifice ancien, lentement construit par les stoïciens (physique, éthique, dialectique) était lié à la rhétorique et à la grammaire, et c'est lui qui a structuré ensuite l'ensemble de la paideia médiévale et moderne, dont la forme accomplie est le modèle des arts libéraux. Le sommet de cette éducation était la dialectique, l'art de l'argument et du débat, mais surtout la méthode de la science.

Ce point me semble majeur : Cicéron, pour ne parler que de lui, n'a cessé dans ses ouvrages de vanter le point de vue de la raison et la nécessité de la méthode, mēme si rien dans son cuvre ne manifeste qu'il savait en vertu de quoi il fallait le faire. Son ideal de sagesse, entièrement commandé par le point de vue moral stoïcien de la nécessité et du calme intérieur, a néanmoins relayé les grandes convictions fondatrices de Platon et des Présocratiques sur la nature. Cicéron est l'exemple d'un penseur qui accepte le primat résolument mythique et pratique de la vision du monde dans laquelle il se trouve, mais qui ne se résout pas à annuler le primat théorique de la vision grecque. La conséquence en est pour lui que le primat de la théologie romaine est finalement un primat de surface, une tradition respectée, mais qui doit se plier devant les exigences de la raison. Voilà l'enseignement des Tusculanes et le sens de l'hommage qui y est rendu à Platon.

Des notions aussi importantes que celles du destin, de la providence, du règne des puissances surnaturelles, toutes notions qui avaient été critiquées et, dans certains cas, réinterprétées par la pensée grecque, risquaient dans la vision romaine pratique du monde de devenir dominantes. Elles l'étaient en fait dans la culture populaire et même dans certaines sagesses sectaires, et le christianisme qui entre en dialogue avec la religion impériale dès le $\mathrm{II}^{\mathrm{e}}$ siècle va beaucoup faire pour transformer cette dramaturgie romaine du règne des dieux et de la Fortune en cadre conceptuel pour le mythe d'une surnature rédemptrice. Nous avons quelques textes qui sont les témoins de cet affrontement, par exemple le Dialogue avec Tryphon de l'apologiste Justin, ou encore les dialogues de jeunesse de saint Augustin. Ces textes montrent que le triomphe de la raison grecque sur le règne des puissances mythiques, même s'il n'était pas considéré comme définitif ou total, paraissait une valeur à transmettre. Plusieurs chrétiens ont lutté contre le rationalisme, aucun n'a réussi à l'évacuer. La majorité, de Clément d'Alexandrie à Augustin, ont plutōt cherché à construire un cadre épistémologique dans lequel la foi pouvait entrer en rapport avec la raison grecque sans chercher à l'annuler. 
L'Antiquité païenne tardive a été aussi fascinée par le mythe. La puissance de la mythologie gréco-romaine, influencèe par les éléments repris des religions orientales dans l'Empire, a été très forte dans l'Antiquité post-classique, autant à Rome que dans l'Ëgypte ptolémaïque. On peut se demander à ce stade-ci de notre réflexion sur quoi se fondait la force du mythe pour que la révolution rationnelle d'Athènes ne devienne pas d'emblèe le paradigme intégrateur de tous les savoirs. Le mythe, certes, transmet un savoir, il structure une vision du monde, mais dans son regard sur le monde, cette vision est essentiellement pratique. Par exemple, la vision d'un salut. Que veut-on dire ici en effet par vision pratique ? La réponse est romaine : on veut dire une pensée dont le telos implicite est toujours déjà une action à faire, une décision à prendre, un sens à interpréter. Ici encore, l'attitude grecque se dèmarque par l'extraordinaire coupure qui sépare dès sa fondation la theoria et la praxis. Si le privilège de la theoria est si exorbitant, c'est précisément parce que la recherche sur la phusis ne possède d'emblée aucune orientation technique ou morale. La réflexion sur la raison pratique, sur cette phronesis si obscure qui, des réflexions de Socrate dans sa prison aux conseils de l'Éthique à Nicomaque, est d'emblée séparée de la theoria. Elle ne peut pretendre au même statut de la philosophie que ce regard désinteressé, cet étonnement, cette critique du mythe qui sont le point de départ de la pensée grecque. Et pourtant, on l'a dit tantôt, tout montre qu'elle lui appartient d'emblée, qu'elle est l'autre face de la raison.

Husserl parle à cet égard d'une epoche de tout intérêt pratique, aussi bien de toute représentation mythique et doxique. Par l'exercice de la philosophie, le Grec de culture (quelle que soit sa nation d'appartenance) cherche une vérité qui ne soit pas la "vérité quotidienne prise dans les liens de la tradition, mais une vérité qui vaille identiquement pour tous ceux que la traditionnalité n'aveugle plus, une vérité en soi $^{11}$. "Le projet d'une science de la nature est donc également, que ce soit sur le plan théorique ou pratique, un affrontement avec le mythe.

L'expérience de la culture grecque offre donc, si on la compare au monde de Rome et de Byzance, une hiérarchie très claire et très nette de la sophia et de la phronesis, hiérarchie dans laquelle le moment fondateur de la pensée grecque s'est investi entièrement. Toute l'histoire ultérieure de la philosophie a retenu ce clivage de la rationalité comme trait déterminant de la raison. Par ce clivage s'illustre en effet la conviction que la raison est à l'œuvre également contre le pouvoir du mythe dans la représentation du monde et dans la conduite de l'existence humaine. Dans tous les cas où la raison demeure muette, dans tous ces cas où la communauté elle-mème s'éloigne de ses convictions rationnelles pour se raccrocher à son symbolisme traditionnel, elle ne renonce jamais à son attitude critique universelle. Le pouvoir du mythe ancien n'a donc été finalement qu'un pouvoir pratique, un pouvoir qui a profité de la disjonction entre la raison théorique et la raison pratique.

11. E. Husserl, La crise des sciences européennes et la phénoménologie transcendantale, p. 366. 
Cet argument nous renvoie à un motif profondément socratique. " Depuis Socrate, écrit encore Husserl, l'homme est devenu un thème dans son humanitê spécifique. "Rien, en effet, chez lui qui soit assujetti aux mythes et aux dieux, rien qui ne réponde à cette voix intérieure de la conscience. Or, précisément cette voix intérieure, dont il tient son mandat, sa tâche propre de philosophie, lui vient de l'Oracle de l'Apollon de Delphes. On a souvent fait valoir par la suite, dans une tradition exégétique qui lie le moment delphique à la révélation de Jésus, comment l'exercice de la raison répondait à une prescription religieuse, une forme d'inspiration qui est à la fois l'endeçà et l'au-delà de la métaphysique platonicienne. Quel est en fait l'équilibre pré-chrétien qui régit ce rapport de la mission de la raison à son origine et à sa finalité ? Dans ses deux traitès sur l'oracle delphique, Plutarque est revenu beaucoup plus tard sur cette question : pourquoi les dieux ne parlent-ils plus, si ce n'est parce que la raison a envahi leurs sanctuaires? II serait trop long d'entrer ici dans le détail des mutations qui ont affecté, entre le $V^{e}$ siècle de Periclès et le monde romain, le rapport de la raison au mythe. En conclusion de ce deuxième moment de mon exposé, je crois seulement utile d'insister sur le fait que l'origine delphique de la conscience, tout comme les mythes orphiques de la connaissance, possédaient dans le monde grec et dans le monde chrétien un sens entièrement différent.

Je termine sur ce point. Dans la pensée grecque, le mythe est un au-delà de la raison qui est en mème temps un défaut de vérité, une sorte de manque ; on dirait, plus exactement, que la vérité du mythe est fautive ou même négative. Le récit archaïque, les généalogies, les chutes et les apocalypses, les cosmologies ne sont jamais que des figures d'une vérité à conquérir, et à conquérir infiniment, par le travail de la raison. Platon lui-mème aurait préféré pouvoir donner une explication rationnelle de la misère de l'aveuglement humain dans la caverne ou de l'origine de l'àme incarnée. Tout ce qui dans la métaphysique hérite du mythe archaïque une forme narrative, une histoire du sens et de la fin, tout cela est conçu comme un échec ou une lacune de la raison. Qu'on lise le Timée ou Empédocle, qu'on relise les stoïciens, on verra combien la raison voudrait exprimer l'ineffable de l'origine ou même la norme de l'action. Mais elle ne le peut pas, elle doit en déporter l'exercice dans cette tâche infinie dont parlait Husserl.

L'exposé chrétien du mystère de la rédemption refuse par contre au mythe archaique ce statut d'emblème métaphorique ou allégorique de la vérité, il propose en lieu et place un récit historique du surnaturel qui se présente comme un récit de la vérité. Dans un langage moderne, on dirait que le mythe chrétien se présente comme véritê. Ce mythe ne doit jamais être critiqué, il ne peut être qu'interprété. On voit où se situe la différence, c'est dans cette prégnance de sens qui, liant l'Ancien et le Nouveau Testament et récapitulant aussi bien Apollon que Dionysos, va disqualifier le mythe grec au profit de la vérité du mystère chrétien. Ces notions sont complexes et leur rapport à la raison n'est pas du mème ordre, il n'est pas isomorphe : là où la raison grecque travaille toujours plus à la critique du mythe, conçu comme forme traditionnelle et nationale, de la doxa, et donc comme fiction recouvrant le non-savoir, devant le mystère chrétien 
elle est appelée à une forme de silence, fait de respect et de désir d'interprèter. La notion d'une intelligence de la foi, cogitatio fidei, s'articule d'emblée sur l'exercice de la théologie et c'est ce nouvel équilibre, en rupture complète avec l'attitude grecque, qui caractérise la culture médiévale.

N'est-il pas intéressant, voire nécessaire, de proposer à l'étudiant contemporain, plus souvent quautrement déchristianisé, de retourner un moment dans l'histoire de la culture, à ce carrefour où les religions monothèistes n'ont pas encore affirmé leur domination (pour ne rien dire du monde oriental structuré par une autre vision du monde), pour y saisir le projet entièrement séculier, laïque et universel d'une raison en butte à l'opinion, en butte aux représentations particulières, en butte au mythe ? Nos étudiants, dont la plupart n'ont reçu aucune éducation religieuse suffisante pour présenter un symbolisme alternatif à la dérive ambiante, et notamment au déclin du symbolisme chrétien, ne peuvent pas comprendre ce que veut dire raison ou mème pluralisme des visions du monde s'ils ne sont pas confrontés à ce moment universel de la raison grecque, préalable au déploiement des monothéismes. J'accorde personnellement beaucoup d'importance à l'éducation religieuse, je pense que tous les élèves du secondaire devraient recevoir une introduction à l'histoire des religions qui poserait les fondements de la tradition monothéiste et montrerait le développement successif du judaïsme, du christianisme et de l'islam. Le retour à la raison grecque, dans son moment socratique, fait de contestation dialectique en même temps que de soumission au non-savoir delphique, me semblerait le meilleur moyen de donner à chacun les moyens de prendre les décisions qui seront les siennes dans le choix de la raison et la considération de l'expérience religieuse.

Je termine cet exposé en faisant retour sur la figure de Socrate, que j'èvoque avec vous dans la foulée des textes que Hegel et Husserl lui ont consacrès. Il serait possible de considerer Socrate comme un personnage, une figure s'agitant au seuil de l'histoire de la pensée occidentale et l'engageant sur un chemin qui vient jusqu'à nous. Nous en ferions un type archaïque, manifestant le travail de l'esprit et la transformation de l'existence dans une forme spirituelle primitive. Il serait également possible de chercher à transcrire sa méthode, son questionnement dans un travail herméneutique semblable à celui de Platon, mais ajusté aux impératifs de notre modernité. Sa conversation serait le modele de toute connaissance de soi. Ces deux efforts ont été faits à notre siècle, le premier par Karl Jaspers, le second par Hans-Georg Gadamer. En dépit de leur grande richesse philosophique, ces efforts demeurent inachevés, car ils ne donnent pas un accès plein et entier à ce qui a été la décision de la pensée grecque, son geste inaugural : en le présentant non seulement comme prophète exemplaire ou comme forme concrète de la conscience, mais comme universel singulier, comme type indépassable de tout questionnement humain, je pense que nous pouvons ouvrir un chemin pour nos étudiants, envers qui nous avons la tâche de déclencher la décision de ce questionnement pour le reste de leur vie. Les premiers poèmes des physiciens, les dialogues platoniciens, les recherches aristotéliciennes, les maximes stoïciennes constituent des formes 
exemplaires de cette sagesse à la fois théorique et pratique que nous appelons " philosophie ". Mieux que quiconque, Husserl a décrit l'avènement de cette attitude universelle :

L'Homme se trouve saisi par la passion d'une considération et d'une connaissance du monde qui se détourne de tous les intérèts pratiques et qui, dans le cercle fermé de son activité de connaissance et des moments à elle consacrés, ne produit ni ne désire rien d'autre que la pure theoria. En d'autres termes, l'homme devient un spectateur désintéressé, un regard jeté sur le monde. il devient philosophe ; ou plutôt sa vie acquiert à partir de là une sensibilité pour des motivations qui ne sont possibles que dans cette attitude : elle est motivee pour des buts et des methodes de pensée d'un nouveau genre, dans lesquels en définitive advient la philosophie et dans lesquels lui-même devient philosophe.

Notre enseignement sera réussi - et on tremble à la pensée que chaque professeur de philosophie fasse chaque fois dans sa classe ce projet du désintéressement - si, comme l'écrit Husserl, s'approchant du telos de la raison, l'existence s'en trouve affectée, modifiée, transformée. Est-ce réactionnaire que de proposer cela?

Département de philosophie Université du Québec à Montréal 